\title{
Un théorème de semi-continuité pour l'entropie des applications méromorphes
}

\author{
Henry de Thélin
}

\begin{abstract}
Résumé
Nous montrons un théorème de semi-continuité supérieure pour l'entropie métrique des applications méromorphes.
\end{abstract}

\begin{abstract}
We prove a theorem of uppersemicontinuity for the metric entropy of meromorphic maps.

Mots-clefs : dynamique complexe, entropie.

Classification : 32U40, 32H50.
\end{abstract}

\section{Introduction}

Soit $X$ une variété $C^{\infty}$ et $f: X \longrightarrow X$ une application $C^{\infty}$. On notera $\mathcal{M}$ l'ensemble des probabilités de $X$ invariantes par $f$. Pour $\mu \in \mathcal{M}, h_{\mu}(f)$ désignera son entropie métrique.

Newhouse a montré dans ce contexte (voir [12]) que l'application $\mu \longrightarrow h_{\mu}(f)$ est semicontinue supérieurement. Pour montrer ce résultat, il définit une notion d'entropie locale qui mesure en quelque sorte le défaut de semi-continuité supérieure, puis il montre que cette entropie est nulle en utilisant le théorème de Yomdin (voir [14]).

L'objectif de cet article est de généraliser ce résultat pour les applications méromorphes.

Soit donc $X$ une variété complexe compacte de dimension $k$ et $f: X \longrightarrow X$ une application méromorphe dominante. On notera $I$ l'ensemble d'indétermination de $f$.

Lorsqu'une probabilité $\mu$ ne charge pas $I$, on peut définir le poussé en avant $f_{*} \mu$, et on dira que $\mu$ est invariante par $f$ si $f_{*} \mu=\mu$. On notera $\mathcal{M}$ l'ensemble de ces mesures.

Pour $\mu$ dans $\mathcal{M}$, on peut définir son entropie métrique $h_{\mu}(f)$. Le but de cet article est de donner des résultats de semi-continuité sur ces entropies métriques. L'application $f$ est holomorphe (donc $C^{\infty}$ ) en dehors de $I$ : la difficulté viendra donc de la présence de l'ensemble d'indétermination $I$ sur lequel $f$ n'est même pas une application. Pour résoudre cette difficulté, l'idée est d'ajouter des hypothèses d'intégrabilité de la fonction log $\operatorname{dist}(x, I)$ 
pour les mesures que l'on considère. Cela permet d'avoir des estimées sur le comportement de $f$ près de $I$.

Plus précisément, le théorème principal est

Théorème 1. Soit $\left(\mu_{n}\right)$ une suite de $\mathcal{M}$ qui converge vers $\mu$ telle que

$$
(H): \lim _{n \rightarrow+\infty} \int \log \operatorname{dist}(x, I) d \mu_{n}(x)=\int \log \operatorname{dist}(x, I) d \mu(x)>-\infty .
$$

Alors

$$
\limsup _{n \rightarrow+\infty} h_{\mu_{n}}(f) \leq h_{\mu}(f) .
$$

Lorsque $f$ est holomorphe (c'est-à-dire $I=\emptyset$ ), on prend $\operatorname{dist}(x, I)$ identiquement égal à 1 dans l'énoncé de sorte que la condition $(H)$ se réduit au fait que la suite $\left(\mu_{n}\right)$ converge vers $\mu$.

L'idée pour démontrer le théorème est de construire des partitions adaptées aux applications méromorphes (comme dans [2]). Ensuite on définira une notion d'entropie locale et enfin en utilisant la généralisation du théorème de Yomdin faite dans [2], nous verrons que cette entropie locale est nulle et nous obtiendrons le théorème.

\section{Partitions adaptées aux applications méromorphes et en- tropie métrique}

Dans un premier temps, nous rappelons la construction de partitions qui permettent de traiter l'entropie des applications méromorphes (voir [2]).

Soit $\eta$ une fonction continue définie sur $X$ comprise entre 0 et 1 et qui vaut 0 sur l'ensemble d'indétermination $I$ de $f$. On a (voir le lemme 2 de [9] et le paragraphe 2.3.4 de [2]) :

Proposition 2. (Mañé)

On peut construire une partition dénombrable $\mathcal{P}$ de $X \backslash\{\eta=0\}$ telle que :

1. Si $x \in X \backslash\{\eta=0\}$, alors diam $\mathcal{P}(x)<\eta(y)$ pour tout $y \in \mathcal{P}(x)$ (ici $\mathcal{P}(x)$ est l'atome de la partition qui contient $x$ ).

2. Pour toute probabilité $\nu$ telle que $\int \log \eta(x) d \nu(x)>-\infty$, on a $H_{\nu}(\mathcal{P})<+\infty$. Ici $H_{\nu}(\mathcal{P})$ désigne l'entropie métrique de la partition $\mathcal{P}$ pour la mesure $\nu$.

Rappelons la forme des éléments de $\mathcal{P}$ car cela nous sera utile.

Tout d'abord, il existe $C>0$ et $r_{0}>0$ telles que pour $0<r \leq r_{0}$, on a une partition $\mathcal{P}_{r}$ de $X$ dont les éléments ont un diamètre inférieur à $r$ et telle que le nombre d'éléments de la partition $\left|\mathcal{P}_{r}\right|$ est plus petit que $C(1 / r)^{2 k}$.

Maintenant, on définit $V_{n}:=\left\{x, e^{-(n+1)}<\eta(x) \leq e^{-n}\right\}$ pour $n \geq 0$. Comme la fonction $\eta$ est comprise entre 0 et 1 , on a $X \backslash\{\eta=0\}=\cup_{n \geq 0} V_{n}$. 
Soit $\mathcal{P}$ la partition définie de la façon suivante : pour $n$ fixé, on considère les ensembles $Q \cap V_{n}$ pour $Q \in \mathcal{P}_{r_{n}}$ avec $r_{n}=e^{-(n+1)}$. Cela définit une partition de $V_{n}$. Maintenant, on obtient la partition $\mathcal{P}$ de $X \backslash\{\eta=0\}$ en prenant tous les $n$ compris entre 0 et $+\infty$. On peut voir que cette partition vérifie les deux points de la proposition (voir le lemme 2 de [9] et le paragraphe 2.3.4 de [2]).

Dans la suite, on regardera cette partition restreinte à $\Omega=X \backslash \cup_{i \geq 0} f^{-i}(I)$ (que l'on notera toujours $\mathcal{P}$ ). L'avantage des partitions sur $\Omega$ c'est que les $f^{i}$ sont bien définis. En particulier, on peut définir la partition $f^{-i}(\mathcal{P})$ : ses atomes sont les $f^{-i}(P):=\{x \in$ $\Omega$ avec $\left.f^{i}(x) \in P\right\}$ où les $P$ sont les atomes de $\mathcal{P}$. Comme $f(\Omega) \subset \Omega$, on obtient une partition de $\Omega$. Les mesures $\nu \in \mathcal{M}$ ont une masse 1 sur $\Omega$ : la partie de $X$ que l'on a enlevée est donc négligeable pour elles.

On a maintenant le résultat de continuité suivant :

Proposition 3. Soit $\left(\mu_{n}\right)$ une suite de $\mathcal{M}$ qui converge vers $\mu$ telle que

$$
\lim _{n \rightarrow+\infty} \int \log \eta(x) d \mu_{n}(x)=\int \log \eta(x) d \mu(x)>-\infty .
$$

Si $\mu$ ne charge pas les bords de la partition $\mathcal{P}$, alors pour tout $q \geq 1$

$$
\lim _{n \rightarrow+\infty} H_{\mu_{n}}\left(\bigvee_{i=0}^{q-1} f^{-i} \mathcal{P}\right)=H_{\mu}\left(\bigvee_{i=0}^{q-1} f^{-i} \mathcal{P}\right) .
$$

Pour démontrer cette proposition, nous aurons besoin du lemme suivant :

Lemme 4. Sous les mêmes hypothèses que dans la proposition précédente, pour $i, j=$ $0, \ldots, q-1$, on $a$

$$
0 \geq \int_{\left\{\eta \circ f^{i} \leq \epsilon\right\}} \log \eta \circ f^{j} d \mu_{n} \geq-\delta(\epsilon)
$$

si $n$ est assez grand. Ici $\delta(\epsilon)$ tend vers 0 quand $\epsilon$ converge vers 0.

De plus, on a le même résultat avec $\mu$ à la place de $\mu_{n}$.

Démonstration. On fait la preuve pour $\mu_{n}$. C'est essentiellement la même pour $\mu$.

Tout d'abord montrons le lemme pour $i=j=0, \ldots, q-1$. On a grâce à l'invariance de $\mu_{n}$

$$
\int_{\left\{\eta \circ f^{j} \leq \epsilon\right\}} \log \eta \circ f^{j}(x) d \mu_{n}(x)=\int_{\{\eta \leq \epsilon\}} \log \eta(x) d \mu_{n}(x) .
$$

Quitte à prendre $\epsilon$ générique de sorte que $\mu$ ne charge pas $\{\eta=\epsilon\}$, la dernière intégrale converge vers $\int_{\{\eta \leq \epsilon\}} \log \eta(x) d \mu(x)$. En effet, d'une part l'hypothèse implique

$$
\lim _{n \rightarrow+\infty} \int \log \eta(x) d \mu_{n}(x)=\int \log \eta(x) d \mu(x)
$$

et d'autre part, $\int_{\{\eta>\epsilon\}} \log \eta(x) d \mu_{n}(x)$ converge vers $\int_{\{\eta>\epsilon\}} \log \eta(x) d \mu(x)$. 
Par convergence dominée, $\int_{\{\eta \leq \epsilon\}} \log \eta(x) d \mu(x)$ tend vers 0 quand $\epsilon$ converge vers 0 . On obtient ainsi l'existence d'une fonction $\delta^{\prime}(\epsilon)$ avec $\lim _{\epsilon \rightarrow 0} \delta^{\prime}(\epsilon)=0$ telle que :

$$
0 \geq \int_{\left\{\eta \circ f^{j} \leq \epsilon\right\}} \log \eta \circ f^{j}(x) d \mu_{n}(x) \geq-\delta^{\prime}(\epsilon)
$$

pour $n$ grand.

Maintenant si $i, j=0, \ldots, q-1$, on coupe l'intégrale

$$
\int_{\left\{\eta \circ f^{i} \leq \epsilon\right\}} \log \eta \circ f^{j}(x) d \mu_{n}(x)
$$

en deux parties

$$
\begin{aligned}
& \int_{\left\{\eta \circ f^{i} \leq \epsilon\right\} \cap\left\{\eta \circ f^{j} \leq \delta^{\prime}(\epsilon)\right\}} \log \eta \circ f^{j}(x) d \mu_{n}(x) \\
& +\int_{\left\{\eta \circ f^{i} \leq \epsilon\right\} \cap\left\{\eta \circ f^{j}>\delta^{\prime}(\epsilon)\right\}} \log \eta \circ f^{j}(x) d \mu_{n}(x) .
\end{aligned}
$$

Le premier terme est plus grand que

$$
\int_{\left\{\eta \circ f^{j} \leq \delta^{\prime}(\epsilon)\right\}} \log \eta \circ f^{j}(x) d \mu_{n}(x)
$$

et ce terme est minoré par $-\delta^{\prime}\left(\delta^{\prime}(\epsilon)\right)$ si $n$ est assez grand par la première partie de la preuve. Cette quantité converge vers 0 quand $\epsilon$ tend vers 0 .

Le second terme est plus grand que (si $\epsilon \leq e^{-1}$ et $\left.\delta^{\prime}(\epsilon) \leq 1\right)$

$$
\begin{aligned}
& \int_{\left\{\eta \circ f^{i} \leq \epsilon\right\}} \log \delta^{\prime}(\epsilon) d \mu_{n}(x) \\
& \geq-\log \delta^{\prime}(\epsilon) \int_{\left\{\eta \circ f^{i} \leq \epsilon\right\}} \log \eta \circ f^{i}(x) d \mu_{n}(x) \geq \delta^{\prime}(\epsilon) \log \delta^{\prime}(\epsilon)
\end{aligned}
$$

si $n$ est suffisamment grand en utilisant le début de la preuve. Cette quantité converge aussi vers 0 quand $\epsilon$ tend vers 0 .

Passons maintenant à la démonstration de la proposition.

Démonstration. Pour un multi-indice $s=\left(s_{0}, \ldots, s_{q-1}\right) \in \mathbb{N}^{q}$, on note $W_{s}=V_{s_{0}} \cap \cdots \cap$ $f^{-q+1} V_{s_{q-1}}$. Lorsque $P \in \bigvee_{i=0}^{q-1} f^{-i} \mathcal{P}$, alors $P$ est dans un des $W_{s}$ pour un certain multiindice $s$ par construction de la partition $\mathcal{P}$. De plus, le nombre de $P \in \bigvee_{i=0}^{q-1} f^{-i} \mathcal{P}$ qui sont dans $W_{s}$ est majoré par $C^{q} e^{2 k q} e^{2 k|s|}=C_{0}^{q} e^{2 k|s|}$ avec $|s|=s_{0}+\cdots+s_{q-1}$. 
Maintenant, pour $A \in \mathbb{N}^{*}$, on a

$$
H_{\mu_{n}}\left(\bigvee_{i=0}^{q-1} f^{-i} \mathcal{P}\right)=\sum_{s \in \mathbb{N}^{q}} \sum_{P \in \bigvee_{i=0}^{q-1}} \sum_{f^{-i} \mathcal{P}, P \subset W_{s}}-\mu_{n}(P) \log \mu_{n}(P)
$$

que l'on divise en :

$$
\sum_{|s| \geq A} \sum_{P \in \bigvee_{i=0}^{q-1}}-\mu_{n}(P) \log \mu_{n}(P)+\sum_{|s|<A} \sum_{P \in \bigvee_{i=0}^{q-1}} \sum_{f^{-i} \mathcal{P}, P \subset W_{s}}-\mu_{n}(P) \log \mu_{n}(P) .
$$

On commence avec le premier terme. En utilisant l'inégalité

$$
-\sum_{i=1}^{m_{0}} x_{i} \log x_{i} \leq\left(\sum_{i=1}^{m_{0}} x_{i}\right)\left(\log m_{0}-\log \sum_{i=1}^{m_{0}} x_{i}\right)
$$

on a

$$
\begin{aligned}
& R_{1}(A)=\sum_{|s| \geq A} \sum_{P \in \bigvee_{i=0}^{q-1}} f_{f^{-i} \mathcal{P}, P \subset W_{s}}-\mu_{n}(P) \log \mu_{n}(P) \\
& \leq \sum_{|s| \geq A} \mu_{n}\left(W_{s}\right)\left(\log \left(\#\left\{P \in \bigvee_{i=0}^{q-1} f^{-i} \mathcal{P}, P \subset W_{s}\right\}\right)-\log \mu_{n}\left(W_{s}\right)\right) \\
& \leq \log C_{0}^{q} \sum_{|s| \geq A} \mu_{n}\left(W_{s}\right)+2 k \sum_{|s| \geq A}|s| \mu_{n}\left(W_{s}\right)+\sum_{|s| \geq A} \mu_{n}\left(W_{s}\right) \log \frac{1}{\mu_{n}\left(W_{s}\right)}
\end{aligned}
$$

Maintenant, en utilisant le lemme 2.3.5 de [2], on obtient

$$
R_{1}(A) \leq C(q) e^{-\frac{A}{2 q}}+\left(\log C_{0}^{q}+2 k+1\right) \sum_{|s| \geq A}|s| \mu_{n}\left(W_{s}\right)
$$

Sur $W_{s}$, on a $-\sum_{j=0}^{q-1} \log \eta \circ f^{j} \geq|s|$. Par ailleurs les $W_{s}$ sont deux à deux disjoints et

$$
\left.\cup_{|s| \geq A} W_{s} \subset \cup_{i=0}^{q-1} \cup_{\left\{s \in \mathbb{N}^{q}\right.}, s_{i} \geq \frac{A}{q}\right\} W_{s} \subset \cup_{i=0}^{q-1}\left\{\eta \circ f^{i} \leq e^{-\frac{A}{q}}\right\},
$$

d'où,

$$
\begin{aligned}
& R_{1}(A) \leq C(q) e^{-\frac{A}{2 q}}+\left(\log C_{0}^{q}+2 k+1\right) \sum_{j=0}^{q-1} \sum_{|s| \geq A} \int_{W_{s}}-\log \eta \circ f^{j}(x) d \mu_{n}(x) \\
& \leq C(q) e^{-\frac{A}{2 q}}+\left(\log C_{0}^{q}+2 k+1\right) \sum_{j=0}^{q-1} \sum_{i=0}^{q-1} \int_{\left\{\eta \circ f^{i}(x) \leq e^{\left.-\frac{A}{q}\right\}}\right.}-\log \eta \circ f^{j}(x) d \mu_{n}(x)
\end{aligned}
$$


qui est aussi petit que l'on veut si on prend $A$ grand puis $n$ grand par le lemme précédent. La même chose est vraie si on remplace $\mu_{n}$ par $\mu$ dans $R_{1}(A)$ : c'est aussi petit que l'on veut si on prend $A$ grand par le lemme précédent.

On considère maintenant le second terme

$$
\sum_{|s|<A} \sum_{P \in \bigvee_{i=0}^{q-1} f^{-i} \mathcal{P}, P \subset W_{s}}-\mu_{n}(P) \log \mu_{n}(P) .
$$

Comme $\mu$ n'a pas de masse sur les bords de la partition cela converge vers

$$
\sum_{|s|<A} \sum_{P \in \bigvee_{i=0}^{q-1}} \sum_{f^{-i} \mathcal{P}, P \subset W_{s}}-\mu(P) \log \mu(P),
$$

quand $n$ tend vers l'infini car il n'y a qu'un nombre fini d'éléments et $\mu_{n}$ converge vers $\mu$.

En conclusion, on a bien que $H_{\mu_{n}}\left(\bigvee_{i=0}^{q-1} f^{-i} \mathcal{P}\right)$ qui converge vers $H_{\mu}\left(\bigvee_{i=0}^{q-1} f^{-i} \mathcal{P}\right)$ quand $n$ tend vers l'infini.

De cette proposition, on en déduit la semi-continuité suivante :

Proposition 5. Soit $\left(\mu_{n}\right)$ une suite de $\mathcal{M}$ qui converge vers $\mu$ telle que

$$
\lim _{n \rightarrow+\infty} \int \log \eta(x) d \mu_{n}(x)=\int \log \eta(x) d \mu(x)>-\infty .
$$

Si $\mu$ ne charge pas les bords de la partition $\mathcal{P}$, alors

$$
\limsup _{n \rightarrow \infty} h_{\mu_{n}}(f, \mathcal{P}) \leq h_{\mu}(f, \mathcal{P})
$$

Démonstration. Soit $\delta>0$.

On choisit d'abord $q \geq 1$ tel que

$$
\left|h_{\mu}(f, \mathcal{P})-\frac{1}{q} H_{\mu}\left(\bigvee_{i=0}^{q-1} f^{-i}(\mathcal{P})\right)\right| \leq \delta .
$$

Ensuite, en utilisant la proposition précédente, on a l'existence de $n_{0}$ avec pour $n \geq n_{0}$

$$
\left|\frac{1}{q} H_{\mu}\left(\bigvee_{i=0}^{q-1} f^{-i}(\mathcal{P})\right)-\frac{1}{q} H_{\mu_{n}}\left(\bigvee_{i=0}^{q-1} f^{-i}(\mathcal{P})\right)\right| \leq \delta
$$

Comme

$$
h_{\mu_{n}}(f, \mathcal{P})=\inf _{q \geq 1} \frac{1}{q} H_{\mu_{n}}\left(\bigvee_{i=0}^{q-1} f^{-i}(\mathcal{P})\right)
$$


on en déduit que pour $n \geq n_{0}$

$$
\begin{aligned}
h_{\mu_{n}}(f, \mathcal{P}) & \leq \frac{1}{q} H_{\mu_{n}}\left(\bigvee_{i=0}^{q-1} f^{-i}(\mathcal{P})\right) \\
& \leq \frac{1}{q} H_{\mu}\left(\bigvee_{i=0}^{q-1} f^{-i}(\mathcal{P})\right)+\delta \leq h_{\mu}(f, \mathcal{P})+2 \delta .
\end{aligned}
$$

Cela démontre la proposition.

\section{Un théorème d'entropie locale à la Newhouse}

Dans cette partie, on considère toujours une fonction $\eta$ continue définie sur $X$ comprise entre 0 et 1 et qui vaut 0 sur l'ensemble d'indétermination $I$. On note $\mathcal{P}$ la partition associée construite au paragraphe précédent. On rappelle que l'on considèrere cette partition restreinte à $\Omega=X \backslash \cup_{i>0} f^{-i}(I)$ (que l'on notera toujours $\mathcal{P}$ ).

Le but de ce paragraphe va être de donner un théorème d'entropie locale à la Newhouse (voir [12]). Commençons par adapter sa définition à notre contexte.

Considérons comme dans [9] les boules dynamiques associée à la fonction $\eta$ : pour $x \in \Omega$,

$$
B(x, \eta, n, f):=\left\{y \in \Omega, \operatorname{dist}\left(f^{i}(x), f^{i}(y)\right) \leq \eta\left(f^{i}(x)\right), i=0, \cdots, n-1\right\} .
$$

Pour $\Lambda$ un ensemble de $X$, notons $r(n, \gamma, \eta, \Lambda, x)$ le cardinal maximal d'un ensemble $(n, \gamma)$-séparé inclus dans $B(x, \eta, n, f) \cap \Lambda$. Si $\nu \in \mathcal{M}$, on définit l'entropie locale

$$
h_{\nu, \eta, l o c}(f):=\lim _{\sigma \rightarrow 1^{-}} \inf _{\{\Lambda, \nu(\Lambda) \geq \sigma\}} \lim _{\gamma \rightarrow 0} \limsup _{n \rightarrow+\infty} \frac{1}{n} \int_{\Lambda} \log r(n, \gamma, \eta, \Lambda, x) d \nu(x) .
$$

L'entropie locale est plus petite que l'entropie topologique de $f$. En particulier, si $X$ est kählérienne elle est majorée par $\max _{l=0, \cdots, k} \log d_{l}$ où les $d_{l}$ sont les degrés dynamiques de $f$ (voir [8], [4] et [5]).

Revenons au cas général où $X$ est une variété complexe compacte de dimension $k$. On a alors

Théorème 6. Pour tout $\nu \in \mathcal{M}$ avec $\int \log \eta(x) d \nu(x)>-\infty$ on a

$$
h_{\nu}(f) \leq h_{\nu}(f, \mathcal{P})+h_{\nu, \eta, l o c}(f) .
$$

Démonstration. Il s'agit ici d'adapter la démonstration de Newhouse (voir [12]).

Soient $N \in \mathbb{N}^{*}$ et $\alpha=\left\{E_{1}, \cdots, E_{r}, X \backslash \cup_{i=1}^{r} E_{i}\right\}$ une partition de $X$ avec les $E_{i}$ compacts. On notera encore $\alpha$ cette partition restreinte à $\Omega$. On prend $\sigma<1$ proche de $1, \Lambda$ un 
ensemble tel que $\nu(\Lambda) \geq \sigma$ puis $\gamma>0$ suffisamment petit pour que $2 \gamma$ soit strictement plus petit que toutes les distances entre $E_{i}$ et $E_{j}$ pour $i \neq j$.

On considère dans la suite $n=q N$ et $\beta=(\Lambda \cap \Omega, \Omega \backslash \Lambda)$. Pour simplifier les expressions nous noterons $\mathcal{P}^{n}=\bigvee_{i=0}^{n-1} f^{-i}(\mathcal{P})$ et $\alpha_{q}=\bigvee_{i=0}^{q-1} f^{-i N}(\alpha)$. On a

$$
H_{\nu}\left(\alpha_{q} \mid \mathcal{P}^{n}\right) \leq H_{\nu}\left(\alpha_{q} \vee \beta \mid \mathcal{P}^{n}\right)=H_{\nu}\left(\beta \mid \mathcal{P}^{n}\right)+H_{\nu}\left(\alpha_{q} \mid \mathcal{P}^{n} \vee \beta\right) \leq \log 2+H_{\nu}\left(\alpha_{q} \mid \mathcal{P}^{n} \vee \beta\right)
$$

Estimons maintenant $H_{\nu}\left(\alpha_{q} \mid \mathcal{P}^{n} \vee \beta\right)$. On a

$$
H_{\nu}\left(\alpha_{q} \mid \mathcal{P}^{n} \vee \beta\right)=\sum_{B \in \mathcal{P}^{n} \vee \beta} \nu(B)\left(-\sum_{A \in \alpha_{q}} \nu(A \mid B) \log \nu(A \mid B)\right) .
$$

On coupe la somme $\sum_{B \in \mathcal{P}^{n} \vee \beta}$ en deux parties : $\sum_{B \in \mathcal{P}^{n} \vee \beta}, B \subset \Lambda$ et $\sum_{B \in \mathcal{P}^{n} \vee \beta}, B \subset \Lambda^{c}$. Commençons par traiter la deuxième partie.

$$
\begin{aligned}
& \sum_{B \in \mathcal{P}^{n} \vee \beta, B \subset \Lambda^{c}} \nu(B)\left(-\sum_{A \in \alpha_{q}} \nu(A \mid B) \log \nu(A \mid B)\right) \leq \nu\left(\Lambda^{c}\right) \log \left(\operatorname{Card}\left(\alpha_{q}\right)\right) \\
& \leq q \nu\left(\Lambda^{c}\right) \log (\operatorname{Card}(\alpha)) \leq q(1-\sigma) \log (\operatorname{Card}(\alpha)) .
\end{aligned}
$$

Pour l'autre terme

$$
\begin{aligned}
& \sum_{B \in \mathcal{P}^{n} \vee \beta, B \subset \Lambda} \nu(B)\left(-\sum_{A \in \alpha_{q}} \nu(A \mid B) \log \nu(A \mid B)\right) \\
= & \int_{\Lambda}-\sum_{A \in \alpha_{q}} \nu(A \mid B(x)) \log \nu(A \mid B(x)) d \nu(x)
\end{aligned}
$$

où $B(x)$ est l'élément $B \in \mathcal{P}^{n} \vee \beta$ qui contient $x$ et où dans la somme à l'intérieur de l'intégrale, on peut se restreindre aux $A \in \alpha_{q}$ qui rencontrent $B(x)$.

Fixons $x \in \Lambda \cap \Omega$ et majorons le terme dans l'intégrale.

On note $F(x)$ un ensemble maximal dans $B(x, \eta, n, f) \cap \Lambda$ tel que si $y, z \in F(x)$ avec $y \neq z$ il existe $0 \leq j \leq q-1$ avec $\operatorname{dist}\left(f^{j N}(y), f^{j N}(z)\right)>\gamma$. C'est en particulier un ensemble $(\gamma, n)$-séparé donc le cardinal de $F(x)$ est plus petit que $r(n, \gamma, \eta, \Lambda, x)$.

Soit $A \in \alpha_{q}$ qui rencontre $B(x)$ et $x_{A} \in A \cap B(x)$. On choisit $\varphi(A) \in F(x)$ tel que $\operatorname{dist}\left(f^{j N}\left(x_{A}\right), f^{j N}(\varphi(A))\right) \leq \gamma$ pour $j=0, \cdots, q-1$. Ce $\varphi(A)$ est bien défini. En effet, on a $B(x) \subset B(x, \eta, n, f) \cap \Lambda$ car si $y \in B(x)$, alors $x$ et $y$ sont dans

$$
\mathcal{P}(x) \cap f^{-1}(\mathcal{P}(f(x))) \cap \cdots \cap f^{-n+1}\left(\mathcal{P}\left(f^{n-1}(x)\right)\right)
$$


et on conclut en utilisant le fait que le diamètre de $\mathcal{P}\left(f^{i}(x)\right)$ est plus petit que $\eta\left(f^{i}(x)\right)$ par la proposition 2 .

Estimons le nombre de $A$ qui ont le même $\varphi(A)$. On rappelle que $\alpha_{q}=\bigvee_{i=0}^{q-1} f^{-i N}(\alpha)$. Si $A, A^{\prime} \in \alpha_{q}$ avec $A \subset f^{-i N}\left(E_{l_{1}}\right)$ et $A^{\prime} \subset f^{-i N}\left(E_{l_{2}}\right)$ où $l_{1} \neq l_{2}$ alors $\varphi(A) \neq \varphi\left(A^{\prime}\right)$ car la distance entre $E_{l_{1}}$ et $E_{l_{2}}$ est plus grande que $2 \gamma$. Il y a donc au plus $2^{q}$ ensembles $A$ qui ont le même $\varphi(A)$.

On a alors :

$$
-\sum_{A \in \alpha_{q}} \nu(A \mid B(x)) \log \nu(A \mid B(x)) \leq \log \left(2^{q} \operatorname{Card}(F(x))\right)
$$

c'est-à-dire

$$
H_{\nu}\left(\alpha_{q} \mid \mathcal{P}^{n} \vee \beta\right) \leq q(1-\sigma) \log (\operatorname{Card}(\alpha))+\int_{\Lambda} \log \left(2^{q} \operatorname{Card}(F(x))\right) d \nu(x) .
$$

En combinant ceci avec l'inégalité obtenue au début de la démonstration, on a

$$
H_{\nu}\left(\alpha_{q} \mid \mathcal{P}^{n}\right) \leq \log 2+q(1-\sigma) \log (\operatorname{Card}(\alpha))+q \log 2+\int_{\Lambda} \log r(n, \gamma, \eta, \Lambda, x) d \nu(x) .
$$

Comme on a pris $n=q N$, on obtient

$\limsup _{q \rightarrow+\infty} \frac{1}{q N} H_{\nu}\left(\alpha_{q} \mid \mathcal{P}^{q N}\right) \leq \frac{(1-\sigma) \log (\operatorname{Card}(\alpha))}{N}+\frac{\log 2}{N}+\limsup _{n \rightarrow+\infty} \frac{1}{n} \int_{\Lambda} \log r(n, \gamma, \eta, \Lambda, x) d \nu(x)$

Maintenant, si on fait tendre $\gamma$ vers 0 , on obtient une inégalité qui est vraie pour tout $\Lambda$ qui vérifie $\nu(\Lambda) \geq \sigma$, d'où :

$$
\begin{aligned}
\limsup _{q \rightarrow+\infty} \frac{1}{q N} H_{\nu}\left(\alpha_{q} \mid \mathcal{P}^{q N}\right) \leq & \frac{(1-\sigma) \log (\operatorname{Card}(\alpha))}{N}+\frac{\log 2}{N} \\
& +\inf _{\{\Lambda, \nu(\Lambda) \geq \sigma\}} \lim _{\gamma \rightarrow 0} \limsup _{n \rightarrow+\infty} \frac{1}{n} \int_{\Lambda} \log r(n, \gamma, \eta, \Lambda, x) d \nu(x) .
\end{aligned}
$$

Enfin, en faisant $\sigma \rightarrow 1$,

$$
\limsup _{q \rightarrow+\infty} \frac{1}{q N} H_{\nu}\left(\alpha_{q} \mid \mathcal{P}^{q N}\right) \leq \frac{\log 2}{N}+h_{\nu, \eta, l o c}(f) .
$$

Il reste à voir comment cette inégalité implique celle que l'on cherche.

Le fait que,

$$
\lim _{q \rightarrow+\infty} \frac{1}{q N} H_{\nu}\left(\bigvee_{i=0}^{q-1} f^{-i N}(\alpha)\right)=\frac{1}{N} h_{\nu}\left(\alpha, f^{N}\right)
$$


et

$$
H_{\nu}\left(\bigvee_{i=0}^{q-1} f^{-i N}(\alpha)\right) \leq H_{\nu}\left(\mathcal{P}^{q N}\right)+H_{\nu}\left(\bigvee_{i=0}^{q-1} f^{-i N}(\alpha) \mid \mathcal{P}^{q N}\right)
$$

impliquent

$$
\frac{1}{N} h_{\nu}\left(\alpha, f^{N}\right) \leq h_{\nu}(f, \mathcal{P})+\frac{\log 2}{N}+h_{\nu, \eta, l o c}(f) .
$$

On utilise ici le fait que l'entropie de $\mathcal{P}$ pour $\nu$ est finie par la proposition 2 et $\int \log \eta(x) d \nu(x)>-\infty$.

Si on prend le sup sur les partitions $\alpha$ puis que l'on fait tendre $N$ vers l'infini, on obtient le théorème.

\section{Théorie de Pesin et applications}

Nous allons voir dans ce paragraphe comment la théorie de Pésin permet de contrôler l'entropie locale précédente.

On munit $X$ d'une famille de cartes $\left(\tau_{x}\right)_{x \in X}$ telles que $\tau_{x}(0)=x, \tau_{x}$ est définie sur une boule $B\left(0, \epsilon_{0}\right) \subset \mathbb{C}^{k}$ avec $\epsilon_{0}$ indépendant de $x$ et la norme de la dérivée première et seconde de $\tau_{x}$ sur $B\left(0, \epsilon_{0}\right)$ est majorée par une constante indépendante de $x$. Pour construire ces cartes il suffit de partir d'une famille finie $\left(U_{i}, \psi_{i}\right)$ de cartes de $X$ et de les composer par des translations.

On considère $\eta$ une fonction continue définie sur $X$ comprise entre 0 et 1 et qui vérifie $\eta(x) \leq \operatorname{dist}(x, I)$. En particulier, elle vaut 0 sur l'ensemble d'indétermination $I$. On rappelle que l'on note :

$$
B(x, \eta, n, f):=\left\{y \in \Omega, \operatorname{dist}\left(f^{i}(x), f^{i}(y)\right) \leq \eta\left(f^{i}(x)\right), i=0, \cdots, n-1\right\} .
$$

On a

Théorème 7. Soit $\nu \in \mathcal{M}$ telle que $\int \log \eta(x) d \nu(x)>-\infty$. Alors

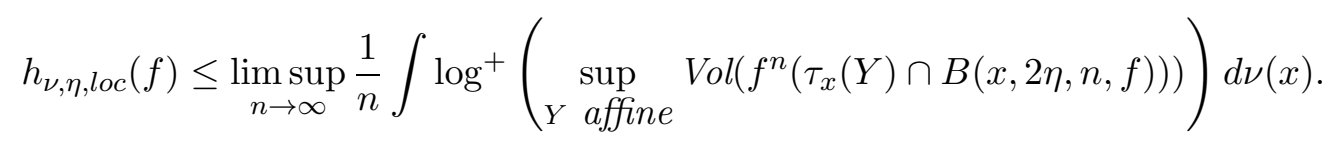

Ici le volume est celui 2l-dimensionnel réel (compté avec multiplicité) où l est la dimension complexe de $Y$ (qui est un plan affine complexe) et $\log ^{+}(a)=\max (0, \log (a))$ pour $a>0$. 


\subsection{Théorème d'Oseledets et transformée de graphe}

Dans ce paragraphe, on considère une probabilité $\nu \in \mathcal{M}$ telle que $\int \log \eta(x) d \nu(x)>$ $-\infty$. Cette hypothèse va permettre de définir des exposants de Lyapounov et de faire de la théorie de Pesin. Rappelons que l'on note $\Omega=X \backslash \cup_{i \geq 0} f^{-i}(I)$. Comme $\nu$ ne charge pas $I, \nu$ est une probabilité de $\Omega$.

Tout d'abord on définit l'extension naturelle :

$$
\widehat{\Omega}:=\left\{\widehat{x}=\left(\cdots, x_{0}, \cdots, x_{n}, \cdots\right) \in \Omega^{\mathbb{Z}}, f\left(x_{n}\right)=x_{n+1}\right\} .
$$

Dans cet espace, $f$ induit une application $\sigma$ qui est le décalage à gauche. Si on note $\pi$ la projection canonique $\pi(\widehat{x})=x_{0}$ alors $\nu$ se relève en une unique probabilité $\widehat{\nu}$ invariante par $\sigma$ qui vérifie $\pi_{*} \widehat{\nu}=\nu$.

Dans toute la suite, on notera $f_{x}=\tau_{f(x)}^{-1} \circ f \circ \tau_{x}$ qui est définie au voisinage de 0 quand $x$ n'est pas dans $I$. Le cocycle auquel nous allons appliquer la théorie de Pesin est alors donné par :

$$
\begin{aligned}
A: \widehat{\Omega} & \longrightarrow M_{k}(\mathbb{C}) \\
\widehat{x} & \longrightarrow D f_{x}(0)
\end{aligned}
$$

où $M_{k}(\mathbb{C})$ est l'ensemble des matrices carrées $k \times k$ à coefficients dans $\mathbb{C}$ et $\pi(\widehat{x})=x$. Afin d'avoir un théorème du type Oseledets, nous aurons besoin du lemme suivant :

\section{Lemme 8.}

$$
\int \log ^{+}\|A(\widehat{x})\| d \widehat{\nu}(\widehat{x})<+\infty
$$

Démonstration. On pose $h(x)=\log ^{+}\left\|D f_{x}(0)\right\|$. On a alors si $\pi(\widehat{x})=x$,

$$
\begin{aligned}
& \int \log ^{+}\|A(\widehat{x})\| d \widehat{\nu}(\widehat{x})=\int h(x) d \widehat{\nu}(\widehat{x}) \\
& =\int h \circ \pi(\widehat{x}) d \widehat{\nu}(\widehat{x})=\int h(x) d \nu(x) .
\end{aligned}
$$

Maintenant comme

$$
\left\|D f_{x}(0)\right\| \leq C^{\prime}\|D f(x)\| \leq C^{\prime \prime} \operatorname{dist}(x, I)^{-p}
$$

(voir le lemme 2.1 de [3]), le lemme découle de l'intégrabilité de la fonction log $\operatorname{dist}(x, I)$ pour la mesure $\nu$.

Grâce à ce lemme, on obtient un théorème du type Oseledets (voir [7] et [13] ainsi que le théorème 2.3 de [11], le théorème 6.1 dans [6] et [10]) : 
Théorème 9. Il existe des réels $\lambda_{1}(\widehat{x})>\lambda_{2}(\widehat{x})>\cdots>\lambda_{l(\widehat{x})}(\widehat{x}) \geq-\infty$, des entiers $m_{1}(\widehat{x}), \cdots, m_{l(\widehat{x})}(\widehat{x})$ et un ensemble $\widehat{\Gamma}$ de mesure pleine pour $\widehat{\nu}$ tels que pour $\widehat{x} \in \widehat{\Gamma}$ on ait une décomposition de $\mathbb{C}^{k}$ de la forme $\mathbb{C}^{k}=\bigoplus_{i=1}^{l(\widehat{x})} E_{i}(\widehat{x})$ où les $E_{i}(\widehat{x})$ sont des sous-espaces vectoriels de dimension $m_{i}(\widehat{x})$. Les fonctions $l(\widehat{x}), \lambda_{i}(\widehat{x})$ et $m_{i}(\widehat{x}) \quad($ pour $i=1, \cdots, l(\widehat{x}))$ sont invariantes par $\sigma$ et on $a$ :

1) $A(\widehat{x}) E_{i}(\widehat{x}) \subset E_{i}(\sigma(\widehat{x}))$ avec égalité si $\lambda_{i}(\widehat{x})>-\infty$.

2) Pour $v \in E_{i}(\widehat{x}) \backslash\{0\}$, on $a$

$$
\lim _{n \rightarrow+\infty} \frac{1}{n} \log \left\|A\left(\sigma^{n-1}(\widehat{x})\right) \cdots A(\widehat{x})\right\|=\lambda_{i}(\widehat{x}) .
$$

Si de plus, $\lambda_{i}(\widehat{x})>-\infty$, on a la même limite quand $n$ tend vers $-\infty$.

Pour tout $\epsilon>0$, il existe une fonction $C_{\epsilon}: \widehat{\Gamma} \longrightarrow G L_{k}(\mathbb{C})$ telle que pour $\widehat{x} \in \widehat{\Gamma}$ :

1) $\lim _{n \rightarrow \infty} \frac{1}{n} \log \left\|C_{\epsilon}^{ \pm 1}\left(\sigma^{n}(\widehat{x})\right)\right\|=0$ (on parle de fonction tempérée).

2) $C_{\epsilon}(\widehat{x})$ envoie la décomposition standard $\bigoplus_{i=1}^{l(\widehat{x})} \mathbb{C}^{m_{i}(\widehat{x})}$ sur $\bigoplus_{i=1}^{l(\widehat{x})} E_{i}(\widehat{x})$.

3) La matrice $A_{\epsilon}(\widehat{x})=C_{\epsilon}^{-1}(\sigma(\widehat{x})) A(\widehat{x}) C_{\epsilon}(\widehat{x})$ est diagonale par bloc $\left(A_{\epsilon}^{1}(\widehat{x}), \cdots, A_{\epsilon}^{l(\widehat{x})}(\widehat{x})\right)$ où chaque $A_{\epsilon}^{i}(\widehat{x})$ est une matrice carrée $m_{i}(\widehat{x}) \times m_{i}(\widehat{x})$ et

$$
\forall v \in \mathbb{C}^{m_{i}(\widehat{x})} \text { on } a e^{\lambda_{i}(\widehat{x})-\epsilon}\|v\| \leq\left\|A_{\epsilon}^{i}(\widehat{x}) v\right\| \leq e^{\lambda_{i}(\widehat{x})+\epsilon}\|v\|
$$

si $\lambda_{i}(\widehat{x})>-\infty$ et

$$
\forall v \in \mathbb{C}^{m_{l(\widehat{x})}(\widehat{x})} \quad\left\|A_{\epsilon}^{l(\widehat{x})}(\widehat{x}) v\right\| \leq e^{\epsilon}\|v\|
$$

si $\lambda_{l(\widehat{x})}(\widehat{x})=-\infty$.

Notons maintenant $g_{\widehat{x}}$ la lecture de $f_{x}$ dans les cartes $C_{\epsilon}$ c'est-à-dire $g_{\widehat{x}}=C_{\epsilon}^{-1}(\sigma(\widehat{x})) \circ$ $f_{x} \circ C_{\epsilon}(\widehat{x})$ où $\pi(\widehat{x})=x$. On a alors

Proposition 10. Il existe des constantes $\epsilon_{1}$ et $C$ qui ne dépendent que de $X$ et $f$ telles que pour $\widehat{x} \in \widehat{\Gamma}$ on ait

1) $g_{\widehat{x}}(0)=0$.

2) $D g_{\widehat{x}}(0)=A_{\epsilon}(\widehat{x})$.

3) Si on note $g_{\widehat{x}}(w)=D g_{\widehat{x}}(0) w+h(w)$, on $a$

$$
\|D h(w)\| \leq 2 C\left\|C_{\epsilon}^{-1}(\sigma(\widehat{x}))\right\|\left\|C_{\epsilon}(\widehat{x})\right\|^{2} \operatorname{dist}(x, I)^{-p}\|w\|
$$

pour $\|w\| \leq \frac{\epsilon_{1} \operatorname{dist}(x, I)^{p}}{\left\|C_{\epsilon}(\widehat{x})\right\|}$.

Démonstration. On suit la preuve de la proposition 8 de [1].

On utilisera (voir le lemme 2.1 de [3])

$$
\|D f(x)\|+\left\|D^{2} f(x)\right\| \leq \operatorname{Cdist}(x, I)^{-p}
$$

et on supposera dans la suite que $p \geq 5$. 
Commençons par montrer que $g_{\widehat{x}}(w)$ est défini pour $\|w\| \leq \frac{\epsilon_{1} \operatorname{dist}(x, I)^{p}}{\left\|C_{\epsilon}(\widehat{x})\right\|}$.

Par construction des cartes $\tau_{x}$, on peut trouver $\epsilon_{0}$ qui ne dépend que de $X$ tel que pour $x \in X$ les $\tau_{x}$ sont définis sur $B\left(0, \epsilon_{0}\right)$ et les $\tau_{x}^{-1} \operatorname{sur} B\left(x, \epsilon_{0}\right)$.

Pour $\|w\| \leq \frac{\epsilon_{0}}{\left\|C_{\epsilon}(\widehat{x})\right\|}$, on a

$$
\begin{aligned}
\operatorname{dist}\left(f \circ \tau_{x} \circ C_{\epsilon}(\widehat{x})(w), f(x)\right) & =\operatorname{dist}\left(f \circ \tau_{x} \circ C_{\epsilon}(\widehat{x})(w), f \circ \tau_{x}(0)\right) \\
& \leq C\left\|C_{\epsilon}(\widehat{x})(w)\right\| \operatorname{dist}\left(\tau_{x}\left(\left[0, C_{\epsilon}(\widehat{x})(w)\right]\right), I\right)^{-p} .
\end{aligned}
$$

L'image par $\tau_{x}$ du segment $\left[0, C_{\epsilon}(\widehat{x})(w)\right]$ vit dans la boule $B\left(x, K\left\|C_{\epsilon}(\widehat{x})(w)\right\|\right)$ où $K$ est une constante qui ne dépend que de $X$. Si $\|w\| \leq \frac{\epsilon_{1} \operatorname{dist}(x, I)^{p}}{\left\|C_{\epsilon}(\widehat{x})\right\|} \leq \frac{\epsilon_{1} \operatorname{dist}(x, I)}{\left\|C_{\epsilon}(\widehat{x})\right\|}$, on a alors

$$
\operatorname{dist}\left(f \circ \tau_{x} \circ C_{\epsilon}(\widehat{x})(w), f(x)\right) \leq C\left(1-K \epsilon_{1}\right)^{-p} \operatorname{dist}(x, I)^{-p}\left\|C_{\epsilon}(\widehat{x})\right\|\|w\| .
$$

Le dernier terme est plus petit que $\epsilon_{0}$ si $\|w\| \leq \frac{\epsilon_{1} \operatorname{dist}(x, I)^{p}}{\left\|C_{\epsilon}(\widehat{x})\right\|}$ (pour un $\epsilon_{1}$ qui ne dépend que de $X$ et $f)$, ce qui signifie que $g_{\widehat{x}}(w)$ est défini pour de tels $w$.

Passons à la preuve de la proposition.

Le point 1 est évident et le point 2 découle du théorème précédent.

Pour le troisième point :

$$
\begin{aligned}
D g_{\widehat{x}}(w)= & D g_{\widehat{x}}(0)+D h(w), \text { d'où pour }\|w\| \leq \frac{\epsilon_{1} \operatorname{dist}(x, I)^{p}}{\left\|C_{\epsilon}(\widehat{x})\right\|} \\
\|D h(w)\| & =\left\|D g_{\widehat{x}}(w)-D g_{\widehat{x}}(0)\right\| \\
& =\left\|C_{\epsilon}^{-1}(\sigma(\widehat{x})) \circ D f_{x}\left(C_{\epsilon}(\widehat{x})(w)\right) \circ C_{\epsilon}(\widehat{x})-C_{\epsilon}^{-1}(\sigma(\widehat{x})) \circ D f_{x}(0) \circ C_{\epsilon}(\widehat{x})\right\| \\
& \leq\left\|C_{\epsilon}^{-1}(\sigma(\widehat{x}))\right\|\left\|D f_{x}\left(C_{\epsilon}(\widehat{x})(w)\right)-D f_{x}(0)\right\|\left\|C_{\epsilon}(\widehat{x})\right\| .
\end{aligned}
$$

Mais on a $\left\|D^{2} f(x)\right\| \leq C d(x, I)^{-p}$ d'où

$$
\begin{aligned}
\|D h(w)\| & \leq C\left\|C_{\epsilon}^{-1}(\sigma(\widehat{x}))\right\|\left\|C_{\epsilon}(\widehat{x})(w)\right\|\left\|C_{\epsilon}(\widehat{x})\right\| \operatorname{dist}\left(\tau_{x}\left(\left[0, C_{\epsilon}(\widehat{x})(w)\right]\right), I\right)^{-p} \\
& \leq C\left(1-K \epsilon_{1}\right)^{-p}\left\|C_{\epsilon}^{-1}(\sigma(\widehat{x}))\right\|\left\|C_{\epsilon}(\widehat{x})\right\|^{2} \operatorname{dist}(x, I)^{-p}\|w\| \\
& \leq 2 C\left\|C_{\epsilon}^{-1}(\sigma(\widehat{x}))\right\|\left\|C_{\epsilon}(\widehat{x})\right\|^{2} \operatorname{dist}(x, I)^{-p}\|w\| .
\end{aligned}
$$

C'est ce que l'on voulait démontrer.

Un dernier théorème que nous utiliserons est la transformée de graphe dans le cas noninversible (voir [6] théorème 6.4). Dans celui-ci $B_{l}(0, R)$ désigne la boule de centre 0 et de rayon $R$ dans $\mathbb{C}^{l}$.

Théorème 11. (transformée de graphe cas non-inversible)(voir théorème 6.4 de [6])

Soient $A: \mathbb{C}^{k_{1}} \longrightarrow \mathbb{C}^{k_{1}}, B: \mathbb{C}^{k_{2}} \longrightarrow \mathbb{C}^{k_{2}}$ des applications linéaires avec $k=k_{1}+k_{2}$. On suppose $A$ inversible, $\|B\|<\left\|A^{-1}\right\|^{-1}$ et on note $\left.\left.\xi=1-\|B\|\left\|A^{-1}\right\| \in\right] 0,1\right]$. Soient $0 \leq \xi_{0} \leq 1,0<\delta<2 \epsilon$ tels que : 


$$
\begin{gathered}
\xi_{0}(1-\xi)+2 \delta\left(1+\xi_{0}\right)\left\|A^{-1}\right\| \leq 1 \text { et } \\
\left(\xi_{0}\|B\|+\delta\left(1+\xi_{0}\right)\right)\left(\left\|A^{-1}\right\|^{-1}-\delta\left(1+\xi_{0}\right)\right)^{-1} \leq \xi_{0} \quad \text { et }
\end{gathered}
$$$$
(\|B\|+2 \delta) e^{-2 \epsilon}+\delta \leq 1 \quad \text { et } \delta\left(1+\xi_{0}\right) \leq \min \left\{\left(\left\|A^{-1}\right\|^{-1}-\xi_{0}\|B\|\right) / 2,\left\|A^{-1}\right\|^{-1}-1\right\} .
$$

Soit $g: B_{k}\left(0, R_{0}\right) \longrightarrow B_{k}\left(0, R_{1}\right)$ holomorphe avec $R_{0} \leq R_{1}, g(0)=0, D g(0)=(A, B)$ et $\|D g(w)-D g(0)\| \leq \delta$ sur $B_{k}\left(0, R_{0}\right)$.

Si $\phi: B_{k_{2}}(0, R) \longrightarrow \mathbb{C}^{k_{1}}$ vérifie $\|\phi(0)\| \leq R^{\prime}$ et $\operatorname{Lip}(\phi) \leq \xi_{0}$ pour un certain $R^{\prime} \leq$ $R \leq R_{0} / 2$ alors il existe $\psi: B_{k_{2}}\left(0, R e^{-2 \epsilon}\right) \longrightarrow \mathbb{C}^{k_{1}}$ avec $\operatorname{Lip}(\psi) \leq \xi_{0},\|\psi(0)\| \leq R^{\prime}$ et $g(\operatorname{graphe}(\psi)) \subset \operatorname{graphe}(\phi)$.

La seule chose qui change par rapport à [6], c'est le fait de mettre $R^{\prime}$ à la place de $R$ à la fin de l'énoncé. C'est une adaptation immédiate de la preuve de [6].

\subsection{Démonstration du théorème 7}

Soit $\nu \in \mathcal{M}$ telle que $\int \log \eta(x) d \nu(x)>-\infty$.

Soit $\delta>0$. Pour $\sigma$ proche de 1 , on a que la différence entre $h_{\nu, \eta, l o c}(f)$ et

$$
\inf _{\{\Lambda, \nu(\Lambda) \geq \sigma\}} \lim _{\gamma \rightarrow 0} \limsup _{n \rightarrow+\infty} \frac{1}{n} \int_{\Lambda} \log r(n, \gamma, \eta, \Lambda, x) d \nu(x)
$$

est plus petite que $\delta$.

Dans un premier temps nous allons construire un ensemble particulier $\Lambda$ sur lequel nous aurons de bonnes estimées. Ensuite nous majorerons $r(n, \gamma, \eta, \Lambda, x)$ sur cet ensemble.

Construction de $\Lambda$ :

On reprend les notations du paragraphe précédent. Tout d'abord, pour $\alpha_{0}>0$ suffisamment petit, la mesure pour $\widehat{\nu}$ de

$$
B_{1}=\left\{\widehat{x} \in \widehat{\Gamma}, \min _{\left\{\lambda_{i}(\widehat{x}) \neq 0\right\}}\left|\lambda_{i}(\widehat{x})\right| \geq \alpha_{0}\right\}
$$

est strictement plus grande que $\sigma$. Ensuite, on fixe $\epsilon>0$ très petit devant $\alpha_{0}$ et $\delta$ et on considère

$$
B_{2}=\left\{\widehat{x} \in \widehat{\Gamma}, \alpha_{1} \leq\left\|C_{\epsilon}(\widehat{x})^{ \pm 1}\right\| \leq \frac{1}{\alpha_{1}}\right\}
$$

Si $\alpha_{1}$ est suffisamment petit on a $\widehat{\nu}\left(B_{1} \cap B_{2}\right)>\sigma$.

Maintenant, si on note 


$$
\mathcal{A}_{n_{0}}:=\left\{x, \forall n \geq n_{0} \min _{i=0, \cdots, n-1} \eta\left(f^{i}(x)\right) \geq e^{-\epsilon n}\right\}
$$

on a

Lemme 12. On a $\nu\left(\cup_{n_{0} \in \mathbb{N}} \mathcal{A}_{n_{0}}\right)=1$.

Démonstration. Par le théorème de Birkhoff, il existe un ensemble $\mathcal{A}$ de mesure 1 pour $\nu$ tel que pour $x \in \mathcal{A}$ on ait :

$$
\lim _{n \rightarrow+\infty} \frac{1}{n} \sum_{i=0}^{n-1} \log \eta\left(f^{i}(x)\right)=\int \log \eta(x) d \nu(x)>-\infty .
$$

Classiquement, cela implique que pour $x \in \mathcal{A}$

$$
\lim _{n \rightarrow+\infty} \frac{1}{n} \log \eta\left(f^{n}(x)\right)=0 .
$$

Soit $x \in \mathcal{A}$. Il existe $n_{1}$ tel que $\frac{1}{n} \log \eta\left(f^{n}(x)\right) \geq-\epsilon$ si $n \geq n_{1}$. On a donc, pour $n>n_{1}$,

$$
\min _{i=n_{1}, \cdots, n-1} \eta\left(f^{i}(x)\right) \geq \min _{i=n_{1}, \cdots, n-1} e^{-\epsilon i} \geq e^{-\epsilon n} .
$$

Maintenant, comme $x \in \mathcal{A}$ les $\eta(x), \cdots, \eta\left(f^{n_{1}}(x)\right)$ sont non nuls et il existe $n_{2}$ tel que pour $n \geq n_{2}$

$$
\min _{i=0, \cdots, n_{1}} \eta\left(f^{i}(x)\right) \geq e^{-\epsilon n} .
$$

Le point $x$ est donc dans $\mathcal{A}_{\max \left(n_{1}, n_{2}\right)}$. Autrement dit $\mathcal{A}$ est inclus dans $\cup_{n_{0} \in \mathbb{N}} \mathcal{A}_{n_{0}}$ et le lemme en découle.

On déduit de ce lemme que $\nu\left(\pi\left(B_{1} \cap B_{2}\right) \cap \mathcal{A}_{n_{0}}\right) \geq \sigma$ si $n_{0}$ est grand. On notera dans la suite $\Lambda=\pi\left(B_{1} \cap B_{2}\right) \cap \mathcal{A}_{n_{0}}$. C'est l'ensemble que l'on voulait construire.

Il s'agit maintenant de majorer

$$
\lim _{\gamma \rightarrow 0} \limsup _{n \rightarrow+\infty} \frac{1}{n} \int_{\Lambda} \log r(n, \gamma, \eta, \Lambda, x) d \nu(x) .
$$

Majoration de $r(n, \gamma, \eta, \Lambda, x)$ sur $\Lambda$ :

Commençons par prendre $\gamma>0$ tel que la différence entre

$$
\limsup _{n \rightarrow+\infty} \frac{1}{n} \int_{\Lambda} \log r(n, \gamma, \eta, \Lambda, x) d \nu(x)
$$

et 


$$
\lim _{\gamma \rightarrow 0} \limsup _{n \rightarrow+\infty} \frac{1}{n} \int_{\Lambda} \log r(n, \gamma, \eta, \Lambda, x) d \nu(x)
$$

soit plus petite que $\delta$.

La majoration de $r(n, \gamma, \eta, \Lambda, x)$ va se faire en deux étapes. Dans un premier temps nous construisons pour $x \in \Lambda$ un ensemble $Q(x)$ qui sera feuilleté par des variétés stables approchées. Ensuite nous utiliserons ces ensembles pour fabriquer un $Y$ affine tel que le volume de $f^{n}\left(\tau_{x}(Y) \cap B(x, 2 \eta, n, f)\right)$ soit essentiellement plus grand que $r(n, \gamma, \eta, \Lambda, x)$.

Etape 1 : construction des $Q(x)$ :

Soit $x \in \Lambda$ et $\widehat{x} \in B_{1} \cap B_{2}$ tel que $\pi(\widehat{x})=x$. On note $E_{u}(\widehat{x})$ la somme directe de tous les $E_{i}(\widehat{x})$ correspondant aux exposants strictement positifs et $E_{s}(\widehat{x})$ la somme directe des $E_{i}(\widehat{x})$ associés aux exposants négatifs ou nuls. La dimension complexe de $E_{u}(\widehat{x})$ sera notée $u$. Elle est comprise entre 0 et $k$.

On se place maintenant dans

$$
C_{\epsilon}^{-1}\left(\sigma^{n}(\widehat{x})\right) E_{u}\left(\sigma^{n}(\widehat{x})\right) \oplus C_{\epsilon}^{-1}\left(\sigma^{n}(\widehat{x})\right) E_{s}\left(\sigma^{n}(\widehat{x})\right)
$$

et on part de

$$
\left\{a_{1}\right\} \times \cdots \times\left\{a_{u}\right\} \times B_{2}\left(0, e^{-4 p \epsilon n}\right)
$$

où $B_{2}\left(0, e^{-4 p \epsilon n}\right)$ est la boule de centre 0 et de rayon $e^{-4 p \epsilon n}$ dans $\mathbb{C}^{k-u}$ et $\left(a_{1}, \cdots, a_{u}\right) \in$ $B_{1}\left(0, e^{-12 p \epsilon n}\right)$ avec $B_{1}\left(0, e^{-12 p \epsilon n}\right)$ la boule de centre 0 et de rayon $e^{-12 p \epsilon n}$ dans $\mathbb{C}^{u}$. Cet ensemble est un graphe $\left(\Phi_{n}(Y), Y\right)$ au-dessus d'une partie de $C_{\epsilon}^{-1}\left(\sigma^{n}(\widehat{x})\right) E_{s}\left(\sigma^{n}(\widehat{x})\right)$ (avec $\left.\Phi_{n}(Y)=\left(a_{1}, \cdots, a_{u}\right)\right)$. Dans le cas où $u=0$ ou $u=k$, tout ceci garde un sens en identifiant $\mathbb{C}^{k}$ avec $\{0\} \times \mathbb{C}^{k}$ ou $\mathbb{C}^{k} \times\{0\}$. Soit $0<\xi_{0}<\frac{1}{2}$ (petit aussi par rapport à $\alpha_{1}$ ).

Lemme 13. Il existe un graphe $\left(\Phi_{n-1}(Y), Y\right)$ au-dessus de

$$
B_{2}\left(0, e^{-4 p \epsilon n-2 \epsilon}\right) \subset C_{\epsilon}^{-1}\left(\sigma^{n-1}(\widehat{x})\right) E_{s}\left(\sigma^{n-1}(\widehat{x})\right)
$$

avec Lip $\Phi_{n-1} \leq \xi_{0}, g_{\sigma^{n-1}(\widehat{x})}\left(\right.$ graphe de $\left.\Phi_{n-1}\right) \subset$ graphe de $\Phi_{n}$ et $\left\|\Phi_{n-1}(0)\right\| \leq e^{-12 p \epsilon n}$.

Démonstration. Par la proposition 10 et le théorème 9, dans le repère

$$
C_{\epsilon}^{-1}\left(\sigma^{n-1}(\widehat{x})\right) E_{u}\left(\sigma^{n-1}(\widehat{x})\right) \oplus C_{\epsilon}^{-1}\left(\sigma^{n-1}(\widehat{x})\right) E_{s}\left(\sigma^{n-1}(\widehat{x})\right),
$$

on peut écrire $g_{\sigma^{n-1}(\widehat{x})}$ sous la forme

$$
g_{\sigma^{n-1}(\widehat{x})}(X, Y)=\left(A_{n-1} X+R_{n-1}(X, Y), B_{n-1} Y+U_{n-1}(X, Y)\right)
$$

avec :

$$
D g_{\sigma^{n-1}(\widehat{x})}(0)=A_{\epsilon}\left(\sigma^{n-1}(\widehat{x})\right)=\left(A_{n-1}, B_{n-1}\right)
$$


où

$$
\left\|A_{n-1}^{-1}\right\|^{-1} \geq e^{\alpha_{0}-\epsilon} \geq e^{2 \epsilon} \text { et }\left\|B_{n-1}\right\| \leq e^{\epsilon} .
$$

En particulier, en utilisant les notations du théorème 11

$$
1-\xi=\left\|B_{n-1}\right\|\left\|A_{n-1}^{-1}\right\| \leq e^{-\epsilon}<1 .
$$

Estimons maintenant le $\delta$ du théorème 11. On a par la proposition 10

$$
\left\|D g_{\sigma^{n-1}(\widehat{x})}(0)-D g_{\sigma^{n-1}(\widehat{x})}(w)\right\| \leq 2 C\left\|C_{\epsilon}^{-1}\left(\sigma^{n}(\widehat{x})\right)\right\|\left\|C_{\epsilon}\left(\sigma^{n-1}(\widehat{x})\right)\right\|^{2} \operatorname{dist}\left(f^{n-1}(x), I\right)^{-p}\|w\|
$$

pour $\|w\| \leq \frac{\epsilon_{1} \operatorname{dist}\left(f^{n-1}(x), I\right)^{p}}{\left\|C_{\epsilon}\left(\sigma^{n-1}(\widehat{x})\right)\right\|}$. Mais comme les fonctions $\left\|C_{\epsilon}^{ \pm 1}\right\|$ sont tempérées et que $\widehat{x} \in B_{2}$, on peut supposer que

$$
\left\|C_{\epsilon}^{-1}\left(\sigma^{n}(\widehat{x})\right)\right\|\left\|C_{\epsilon}\left(\sigma^{n-1}(\widehat{x})\right)\right\|^{2} \leq \frac{1}{\alpha_{1}^{3}} e^{3 \epsilon n} .
$$

Maintenant, comme $x \in \mathcal{A}_{n_{0}}$ et $\eta(x) \leq \operatorname{dist}(x, I)$, on a $\operatorname{dist}\left(f^{n-1}(x), I\right) \geq \eta\left(f^{n-1}(x)\right) \geq$ $e^{-\epsilon n}$ pour $n \geq n_{0}$ et alors

$$
\left\|D g_{\sigma^{n-1}(\widehat{x})}(0)-D g_{\sigma^{n-1}(\widehat{x})}(w)\right\| \leq \frac{2 C}{\alpha_{1}^{3}} e^{3 \epsilon n} e^{p \epsilon n}\|w\| \leq e^{2 p \epsilon n}\|w\|
$$

si $\|w\| \leq e^{-2 p \epsilon n} \leq \frac{\alpha_{1} \epsilon_{1} e^{-p \epsilon n}}{e^{\epsilon n}} \leq \frac{\epsilon_{1} \operatorname{dist}\left(f^{n-1}(x), I\right)^{p}}{\left\|C_{\epsilon}\left(\sigma^{n-1}(\widehat{x})\right)\right\|}$ pour $n$ grand.

En particulier, pour $\|w\| \leq R_{0}=e^{-3 p \epsilon n}$ et $n$ grand, le $\delta$ du théorème 11 est plus petit que $e^{-p \epsilon n}$.

Le lemme découle alors de la proposition 11 en prenant $R=e^{-4 p \epsilon n}$ et $R^{\prime}=e^{-12 p \epsilon n}$ avec $n$ grand. Ici le $n$ grand est indépendant du $x$ choisi dans $\Lambda$.

Maintenant on recommence ce que l'on vient de faire avec $g_{\sigma^{n-2}(\widehat{x})}$ au lieu de $g_{\sigma^{n-1}(\widehat{x})}$. On se place toujours dans la boule $B\left(0, R_{0}\right)$ avec $R_{0}=e^{-3 p \epsilon n}$ et on prend $R=e^{-4 p \epsilon n-2 \epsilon}$. On obtient ainsi (toujours pour le même $n$ grand que précédemment) l'existence d'un graphe $\left(\Phi_{n-2}(Y), Y\right)$ au-dessus de

$$
B_{2}\left(0, e^{-4 p \epsilon n-4 \epsilon}\right) \subset C_{\epsilon}^{-1}\left(\sigma^{n-2}(\widehat{x})\right) E_{s}\left(\sigma^{n-2}(\widehat{x})\right)
$$

avec Lip $\Phi_{n-2} \leq \xi_{0}, g_{\sigma^{n-2}(\widehat{x})}\left(\right.$ graphe de $\left.\Phi_{n-2}\right) \subset$ graphe de $\Phi_{n-1}$ et $\left\|\Phi_{n-2}(0)\right\| \leq e^{-12 p \epsilon n}$.

On continue ainsi le procédé. A la fin on obtient un graphe $\left(\Phi_{0}(Y), Y\right)$ au-dessus de

$$
B_{2}\left(0, e^{-4 p \epsilon n-2 \epsilon n}\right) \subset C_{\epsilon}^{-1}(\widehat{x}) E_{s}(\widehat{x})
$$

avec Lip $\Phi_{0} \leq \xi_{0}, g_{\widehat{x}}\left(\right.$ graphe de $\left.\Phi_{0}\right) \subset$ graphe de $\Phi_{1}$ et $\left\|\Phi_{0}(0)\right\| \leq e^{-12 p \epsilon n}$. 
Grâce aux estimées sur $B_{2}$, l'image de ce graphe par $C_{\epsilon}(\widehat{x})$ est un graphe $\left(\Psi_{0}(Y), Y\right)$ dans le repère $E_{u}(\widehat{x}) \oplus E_{s}(\widehat{x})$ au-dessus d'une boule $B_{2}\left(0, e^{-4 p \epsilon n-3 \epsilon n}\right)$. Il vérifie Lip $\Psi_{0} \leq \frac{\xi_{0}}{\alpha_{1}^{2}}$ qui est aussi petit que l'on veut pourvu que $\xi_{0}$ le soit par rapport à $\alpha_{1}$ (ce que l'on a supposé) et $\left\|\Psi_{0}(0)\right\| \leq e^{-12 p \epsilon n+\epsilon n}$.

On notera $Q(x)$ la réunion de tous ces graphes $\left(\Psi_{0}(Y), Y\right)$ quand $\left(a_{1}, \cdots, a_{u}\right)$ décrit $B_{1}\left(0, e^{-12 p \epsilon n}\right)$.

\section{Etape 2 : fin de la majoration :}

Soit $x \in \Lambda$ fixé et $x_{1}, \cdots, x_{N}$ un ensemble maximal de points $(n, \gamma)$-séparés dans $B(x, \eta, n, f) \cap \Lambda$. On supposera dans la suite que $N \geq e^{40 p k \epsilon n}$ (sinon on verra à la fin de ce paragraphe que l'on obtient directement la majoration voulue).

Quitte à remplacer $N$ par $N / K_{0}$ où $K_{0}$ ne dépend que de $X$, on peut supposer que la dimension des $E_{u}\left(\widehat{x_{i}}\right)$ est la même pour tous les $x_{i}$ (on la notera $u$ ) et que tous les $\tau_{x_{i}}$ sont égaux à une seule carte $\psi: U \longrightarrow X$ modulo des translations. En particulier la distance entre le bord de $U$ et les $\psi^{-1}\left(x_{i}\right)$ est plus grande que $\epsilon_{0}$.

Toujours quitte à remplacer $N$ par $N / K_{0}$, on peut supposer que les graphes qui constituent les $\psi^{-1}\left(\tau_{x_{i}}\left(Q\left(x_{i}\right)\right)\right.$ ) (qui sont juste les translatés des $\left.Q\left(x_{i}\right)\right)$ sont des graphes audessus d'un plan complexe $P$ de dimension $k-u$. Par ailleurs comme les $\left\|\Psi_{0}(0)\right\|$ sont très petits devant $e^{-4 p \epsilon n-3 \epsilon n}$, pour chaque $\psi^{-1}\left(\tau_{x_{i}}\left(Q\left(x_{i}\right)\right)\right)$, on peut supposer que tous les graphes qui le constituent sont des graphes au-dessus d'une même boule de rayon $e^{-6 p \epsilon n}$ dans $P$ (quitte à ne garder que cette partie là). Si on note $G\left(x_{i}\right)$ un des graphes qui constitue $\psi^{-1}\left(\tau_{x_{i}}\left(Q\left(x_{i}\right)\right)\right)$, le volume $2(k-u)$-dimensionnel réel de la projection de tous ces graphes $G\left(x_{i}\right)(i=1, \cdots, N)$ est supérieur à $N e^{-12 p k \epsilon n}$.

Notons $\pi_{1}$ la projection orthogonale sur $P$. Comme $\pi_{1}(U)$ vit dans un compact de $P$, on peut trouver un point $a$ de $P$ tel que la fibre $L=\pi_{1}^{-1}(a)$ coupe $\cup_{i=1}^{N} G\left(x_{i}\right)$ en au moins $N e^{-12 p k \epsilon n}$ points (éventuellement divisé par une constante qui ne dépend que de $U$ ). Pour simplifier les notations, nous noterons $x_{i}$ avec $i=1, \cdots, N e^{-12 p k \epsilon n}$ ces points. Par construction, quand $L$ coupe $G\left(x_{i}\right)$ il coupe tous les graphes qui constituent $\psi^{-1}\left(\tau_{x_{i}}\left(Q\left(x_{i}\right)\right)\right)$.

Signalons deux cas particuliers : quand $u=0, L$ est en fait un point commun aux $G\left(x_{i}\right)$ qui eux sont des ellipsoïdes. Lorsque $u=k, L$ est égal à $U$ et les $G\left(x_{i}\right)$ sont des points.

Montrons maintenant que les $\psi^{-1}\left(\tau_{x_{i}}\left(Q\left(x_{i}\right)\right)\right)$ sont deux à deux disjoints. Si $i \neq j$ les points $x_{i}$ et $x_{j}$ sont $(n, \gamma)$-séparés et il existe donc $l$ compris entre 0 et $n-1$ avec $\operatorname{dist}\left(f^{l}\left(x_{i}\right), f^{l}\left(x_{j}\right)\right) \geq \gamma$. D'autre part, on a

Lemme 14. Pour $l=0, \cdots, n-1$ et $i=1, \cdots, N$

$$
f^{l}\left(\tau_{x_{i}}\left(Q\left(x_{i}\right)\right)\right) \subset B\left(f^{l}\left(x_{i}\right), e^{-2 p \epsilon n}\right) .
$$

Démonstration. Par construction, on a pour $l=0, \cdots, n$

$$
g_{\sigma^{l-1}\left(\widehat{x}_{i}\right)} \circ \cdots \circ g_{\widehat{x_{i}}}\left(C_{\epsilon}^{-1}\left(\widehat{x_{i}}\right)\left(Q\left(x_{i}\right)\right)\right) \subset B\left(0, e^{-3 p \epsilon n}\right)
$$

(où par convention $g_{\sigma^{l-1}\left(\widehat{x}_{i}\right)} \circ \cdots \circ g_{\widehat{x}_{i}}$ est l'identité si $l=0$ ). 
De plus,

$$
\begin{aligned}
g_{\sigma^{l-1}\left(\widehat{x_{i}}\right)} \circ \cdots \circ g_{\widehat{x_{i}}} & =C_{\epsilon}^{-1}\left(\sigma^{l}\left(\widehat{x_{i}}\right)\right) \circ f_{f^{l-1}\left(x_{i}\right)} \circ \cdots \circ f_{x_{i}} \circ C_{\epsilon}\left(\widehat{x_{i}}\right) \\
& =C_{\epsilon}^{-1}\left(\sigma^{l}\left(\widehat{x}_{i}\right)\right) \circ \tau_{f^{l}\left(x_{i}\right)}^{-1} \circ f^{l} \circ \tau_{x_{i}} \circ C_{\epsilon}\left(\widehat{x_{i}}\right) .
\end{aligned}
$$

On a donc

$$
f^{l}\left(\tau_{x_{i}}\left(Q\left(x_{i}\right)\right)\right) \subset \tau_{f^{l}\left(x_{i}\right)} \circ C_{\epsilon}\left(\sigma^{l}\left(\widehat{x_{i}}\right)\right)\left(B\left(0, e^{-3 p \epsilon n}\right)\right)
$$

ce qui donne le lemme (toujours pour $n$ grand) grâce au contrôle de $\left\|C_{\epsilon}\left(\widehat{x_{i}}\right)\right\|$ par $1 / \alpha_{1}$, le fait que $C_{\epsilon}$ est tempérée et le contrôle des dérivées premières de $\tau_{x}$ pour $x \in X$.

Le fait que $\operatorname{dist}\left(f^{l}\left(x_{i}\right), f^{l}\left(x_{j}\right)\right) \geq \gamma$ combiné au lemme implique que les $\tau_{x_{i}}\left(Q\left(x_{i}\right)\right)$ sont deux à deux disjoints (et donc les $\psi^{-1}\left(\tau_{x_{i}}\left(Q\left(x_{i}\right)\right)\right)$ aussi).

Dans le cas particulier où $u=0$ cela implique que $N e^{-12 p k \epsilon n}$ est inférieur à 1 ce qui contredit le fait que $N \geq e^{40 p k \epsilon n}$. Dans la suite, on supposera donc que $u>0$.

Pour $i=1, \cdots, N e^{-12 p k \epsilon n}$, l'ensemble $C_{\epsilon}^{-1}\left(\sigma^{n}\left(\widehat{x}_{i}\right)\right) \circ \tau_{f^{n}\left(x_{i}\right)}^{-1}\left(f^{n}\left(\psi(L) \cap \tau_{x_{i}}\left(Q\left(x_{i}\right)\right)\right)\right)$ rencontre par construction tous les

$$
\left\{a_{1}\right\} \times \cdots \times\left\{a_{u}\right\} \times B_{2}\left(0, e^{-4 p \epsilon n}\right)
$$

où $\left(a_{1}, \cdots, a_{u}\right) \in B_{1}\left(0, e^{-12 p \epsilon n}\right)$ (car $L$ coupe tous les graphes qui constituent $\left.\psi^{-1}\left(\tau_{x_{i}}\left(Q\left(x_{i}\right)\right)\right)\right)$. En particulier le volume $2 u$-dimensionnel de $C_{\epsilon}^{-1}\left(\sigma^{n}\left(\widehat{x}_{i}\right)\right) \circ \tau_{f^{n}\left(x_{i}\right)}^{-1}\left(f^{n}\left(\psi(L) \cap \tau_{x_{i}}\left(Q\left(x_{i}\right)\right)\right)\right)$ est minoré par $e^{-24 p k \epsilon n}$. Comme les $x_{i}$ sont dans $\Lambda$, que les $C_{\epsilon}^{ \pm 1}$ sont tempérées, et que les dérivées des $\tau_{y}^{ \pm 1}$ sont bornées, on obtient que le volume de $f^{n}\left(\psi(L) \cap \tau_{x_{i}}\left(Q\left(x_{i}\right)\right)\right)$ est plus grand que $e^{-25 p k \epsilon n}$. Par suite celui de $f^{n}\left(\psi(L) \cap\left(\cup_{i=1}^{N e^{-12 p k \epsilon n}} \tau_{x_{i}}\left(Q\left(x_{i}\right)\right)\right)\right)$ compté avec multiplicité est minoré par $N e^{-40 p k \epsilon n}$ car les $\tau_{x_{i}}\left(Q\left(x_{i}\right)\right)$ sont disjoints.

Maintenant, on a $\cup_{i=1}^{N e^{-12 p k \epsilon n}} \tau_{x_{i}}\left(Q\left(x_{i}\right)\right) \subset B(x, 2 \eta, n, f)$. En effet, soit $y \in \cup_{i=1}^{N e^{-12 p k \epsilon n}} \tau_{x_{i}}\left(Q\left(x_{i}\right)\right)$ et $i$ tel que $y \in \tau_{x_{i}}\left(Q\left(x_{i}\right)\right)$. Comme $x_{i} \in B(x, \eta, n, f)$, on a par le lemme précédent, pour $l=0, \cdots, n-1$

$$
\begin{aligned}
\operatorname{dist}\left(f^{l}(y), f^{l}(x)\right) & \leq \operatorname{dist}\left(f^{l}(y), f^{l}\left(x_{i}\right)\right)+\operatorname{dist}\left(f^{l}\left(x_{i}\right), f^{l}(x)\right) \\
& \leq e^{-2 p \epsilon n}+\eta\left(f^{l}(x)\right) \leq 2 \eta\left(f^{l}(x)\right) .
\end{aligned}
$$

La dernière inégalité provient du fait que $x \in \mathcal{A}_{n_{0}}$.

En combinant ce qui précède on a donc que le volume $2 u$-dimensionnel de $f^{n}(\psi(L) \cap$ $B(x, 2 \eta, n, f))$ est minoré par $N e^{-40 p k \epsilon n}$. L'application $\tau_{x}$ s'écrit $\psi \circ t$ où $t$ est une translation, donc en posant $Y=t^{-1}(L)$, on a

$$
\operatorname{Vol}\left(f^{n}\left(\tau_{x}(Y) \cap B(x, 2 \eta, n, f)\right)\right) \geq N e^{-40 p k \epsilon n}
$$


avec $Y$ affine. Autrement dit

$$
N=r(n, \gamma, \eta, \Lambda, x) \leq \operatorname{Vol}\left(f^{n}\left(\tau_{x}(Y) \cap B(x, 2 \eta, n, f)\right)\right) e^{40 p k \epsilon n} .
$$

Dans le cas où $N<e^{40 p k \epsilon n}$, on a

$$
N=r(n, \gamma, \eta, \Lambda, x) \leq \max \left(\operatorname{Vol}\left(f^{n}\left(\tau_{x}(Y) \cap B(x, 2 \eta, n, f)\right)\right), 1\right) e^{40 p k \epsilon n} .
$$

D'où finalement,

$$
\begin{aligned}
h_{\nu, \eta, l o c}(f)-\delta & \leq \inf _{\{\Lambda, \nu(\Lambda) \geq \sigma\}} \lim _{\gamma \rightarrow 0} \limsup _{n \rightarrow+\infty} \frac{1}{n} \int_{\Lambda} \log r(n, \gamma, \eta, \Lambda, x) d \nu(x) \\
& \leq \limsup _{n \rightarrow+\infty} \frac{1}{n} \int_{\Lambda} \log r(n, \gamma, \eta, \Lambda, x) d \nu(x)+\delta \\
& \leq \limsup _{n \rightarrow+\infty} \frac{1}{n} \int_{\Lambda} \log ^{+}\left(\sup _{Y \text { affine }} \operatorname{Vol}\left(f^{n}\left(\tau_{x}(Y) \cap B(x, 2 \eta, n, f)\right)\right)\right) d \nu(x)+\delta+40 p k \epsilon .
\end{aligned}
$$

C'est ce que l'on voulait démontrer.

\section{Démonstration du théorème}

Soit $m \in \mathbb{N}^{*}$. On définit comme dans [2]

$$
\rho_{m}(x)=\left(\frac{\operatorname{dist}(x, I) \times \cdots \times \operatorname{dist}\left(f^{m-1}(x), I\right)}{K^{m}}\right)^{p} .
$$

La fonction $\rho_{m}$ vaut 0 sur $I$, on peut donc définir pour $\nu \in \mathcal{M}$ la quantité $h_{\nu, \frac{\rho_{m}}{2}, l o c}(f)$ comme au paragraphe 2 . On a alors

Théorème 15. Soit $L>0$. Pour tout $\delta>0$, il existe $m_{0}$ tel que pour $m \geq m_{0}$

$$
\sup _{\left\{\nu \in \mathcal{M}, \int \log d(x, I) d \nu(x) \geq-L\right\}} h_{\nu, \frac{\rho m}{2}, l o c}(f) \leq \delta .
$$

Démonstration. La démonstration de ce théorème repose sur l'inégalité volumique du paragraphe précédent ainsi que la version méromorphe du théorème de Yomdin développée dans la proposition 2.3.2 de [2]. On adoptera ici les notations de cette proposition.

Fixons $L>0$ puis $\delta>0$. On prend $r$ tel que $\frac{1}{r} \log K<\delta$ et $\frac{L}{r}<\delta$. Soit $m_{0}$ tel que $\max _{l=0, \cdots, 2 k}\left(\frac{1}{m} \log (C(X, l, r))\right)<\delta$ pour $m \geq m_{0}$. On considère $\nu \in \mathcal{M}$ telle que $\int \log d(x, I) d \nu(x) \geq-L$.

Comme $\nu$ est invariante par $f$, la fonction $\log \frac{\rho_{m}}{2}$ est intégrable. Si on veut on a $\operatorname{dist}(x, I) \geq \frac{\rho_{m}(x)}{2}$ ce qui implique par le théorème 7 que 


$$
h_{\nu, \frac{\rho m}{2}, l o c}(f) \leq \limsup _{n \rightarrow \infty} \frac{1}{n} \int \log ^{+}\left(\sup _{Y \text { affine }} \operatorname{Vol}\left(f^{n}\left(\tau_{x}(Y) \cap B\left(x, \rho_{m}, n, f\right)\right)\right)\right) d \nu(x) .
$$

On voit que les boules $B\left(x, \rho_{m}, n, f\right)$ sont incluses dans celle $B_{n}(x)$ définies au début du paragraphe 2.3 de [2]. En particulier, par la proposition 2.3.2 de [2], le volume de $f^{n}\left(\tau_{x}(Y) \cap B\left(x, \rho_{m}, n, f\right)\right)$ est majoré par (ici $l$ est la dimension réelle de $Y$ )

$$
C(X, l, r)^{n / m+2 m} \times K^{\frac{2 n p l}{r}+\frac{4 m p l}{r}} \times \prod_{0 \leq i \leq n-1} \operatorname{dist}\left(f^{i}(x), I\right)^{\frac{-4 p l}{r}} .
$$

Si on pose $C(X, r)=\max _{l=0, \cdots, 2 k} C(X, l, r)$, on a donc

$$
\begin{aligned}
& \frac{1}{n} \int \log ^{+}\left(\sup _{Y \text { affine }} \operatorname{Vol}\left(f^{n}\left(\tau_{x}(Y) \cap B\left(x, \rho_{m}, n, f\right)\right)\right)\right) d \nu(x) \\
& \leq \frac{1}{m} \log (C(X, r))+\frac{2 p l}{r} \log K-\frac{4 k p}{r n} \sum_{i=0}^{n-1} \int \log \operatorname{dist}\left(f^{i}(x), I\right) d \nu(x)+\frac{C(X, m, r, K, p)}{n} \\
& \leq \delta+2 k p \delta-\frac{4 k p}{r} \int \log \operatorname{dist}(x, I) d \nu(x)+\frac{C(X, m, r, K, p)}{n} \\
& \leq \delta+2 k p \delta+4 k p \delta+\frac{C(X, m, r, K, p)}{n} .
\end{aligned}
$$

On obtient ainsi

$$
h_{\nu, \frac{\rho_{m}}{2}, l o c}(f) \leq 7 k p \delta
$$

pour tout $\nu \in \mathcal{M}$ telle que $\int \log d(x, I) d \nu(x) \geq-L$.

C'est ce que l'on voulait démontrer.

Passons maintenant à la démonstration du théorème principal.

Démonstration. Démonstration du théorème 1 :

Soit $\left(\mu_{n}\right)$ une suite de $\mathcal{M}$ qui converge vers $\mu$ telle que

$$
(H): \lim _{n \rightarrow+\infty} \int \log d(x, I) d \mu_{n}(x)=\int \log d(x, I) d \mu(x)>-\infty .
$$

Soit $\delta>0$. On prend $L=-\int \log d(x, I) d \mu(x)+1$. Par l'hypothèse (H), il existe $n_{0}$ tel que pour $n \geq n_{0}$ on ait

$$
\int \log d(x, I) d \mu_{n}(x) \geq-L
$$


On peut donc appliquer le théorème précédent à ce $L$ et aux mesures $\mu_{n}$ pour $n \geq n_{0}$ : on obtient ainsi l'existence de $m_{0}$ tel que pour $m \geq m_{0}$

$$
\sup _{n \geq n_{0}} h_{\mu_{n}, \frac{\rho m}{2}, l o c}(f) \leq \delta .
$$

Dans la suite on fixe $m \geq m_{0}$ et on considère $n \geq n_{0}$. Par le théorème 6 , on a

$$
h_{\mu_{n}}(f) \leq h_{\mu_{n}}(f, \mathcal{P})+h_{\mu_{n}, \frac{\rho_{m}}{2}, l o c}(f) \leq h_{\mu_{n}}(f, \mathcal{P})+\delta .
$$

Ici, $\mathcal{P}$ est la partition associée à $\frac{\rho_{m}}{2}$ et quitte à la bouger un peu on peut supposer que $\mu$ ne charge pas ses bords.

L'hypothèse $(\mathrm{H})$ et l'invariance par $f$ des mesures $\mu_{n}$ et $\mu$ impliquent que

$$
\lim _{n \rightarrow+\infty} \int \log \frac{\rho_{m}(x)}{2} d \mu_{n}(x)=\int \log \frac{\rho_{m}(x)}{2} d \mu(x)>-\infty .
$$

En particulier, par la proposition 5 , on a

$$
\limsup _{n \rightarrow+\infty} h_{\mu_{n}}(f) \leq \limsup _{n \rightarrow+\infty} h_{\mu_{n}}(f, \mathcal{P})+\delta \leq h_{\mu}(f, \mathcal{P})+\delta \leq h_{\mu}(f)+\delta .
$$

Cela démontre le théorème. 


\section{Références}

[1] H. De Thélin Sur les exposants de Lyapounov des applications méromorphes, Invent. Math., 172 (2008), 89-116.

[2] H. De Thélin et G. Vigny, Entropy of meromorphic maps and dynamics of birational maps, Mém. Soc. Math. Fr., 122 (2010).

[3] T.-C. Dinh et C. Dupont, Dimension de la mesure d'équilibre d'applications méromorphes, J. Geom. Anal., 14 (2004), 613-627.

[4] T.-C. Dinh et N. Sibony, Regularization of currents and entropy, Ann. Ecole Norm. Sup., 37 (2004), 959-971.

[5] T.-C Dinh et N. Sibony, Une borne supérieure pour l'entropie topologique d'une application rationnelle, Ann. of Math., 161 (2005), 1637-1644.

[6] C. Dupont, Large entropy measures for endomorphisms of $\mathbb{C P}^{k}$, Israel J. Math., 192 (2012), 505-533.

[7] G. Froyland, S. Lloyd et A. Quas, Coherent structures and isolated spectrum for Perron-Frobenius cocycles, Ergodic Theory Dynam. Systems, 30 (2010), 729-756.

[8] M. Gromov, On the entropy of holomorphic maps, Enseign. Math., 49 (2003), 217-235.

[9] R. Mañé, A proof of Pesin's formula, Ergod. Th. Dynam. Syst. 1 (1981), 95-102.

[10] R. Mañé, Lyapounov exponents and stable manifolds for compact transformations, Lecture Notes in Math., 1007 (1983), 522-577.

[11] S. E. Newhouse, Entropy and volume, Ergodic Theory Dynam. Systems, 8 (1988), 283-299.

[12] S. E. Newhouse, Continuity properties of entropy, Ann. of Math., 129 (1989), 215-235.

[13] P. Thieullen, Fibrés dynamiques asymptotiquement compacts. Exposants de Lyapounov. Entropie. Dimension, Ann. Inst. H. Poincaré Anal. Non Linéaire, 4 (1987), 49-97.

[14] Y. Yomdin, Volume growth and entropy, Israël J. Math. 57 (1987), 285-300.

Henry De Thélin, Université Paris 13, Sorbonne Paris Cité, LAGA, CNRS (UMR 7539), F-93430, Villetaneuse, France.

dethelin@math.univ-paris13.fr 